\title{
Complete azimuthal decomposition of optical fields
}

\author{
A. Dudley*a, I. Litvin ${ }^{\mathrm{a}}$, F. S. Roux ${ }^{\mathrm{a}}$ and A. Forbes ${ }^{\mathrm{a}, \mathrm{b}, \mathrm{c}}$

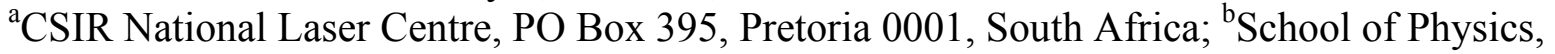 \\ University of KwaZulu-Natal, Private Bag X54001, Durban 4000, South Africa; ${ }^{\text {cStellenbosch }}$ \\ University, Private Bag X1, Matieland 7602, South Africa.
}

\begin{abstract}
By using digital holograms, we present a simple technique for performing a complete azimuthal decomposition of an arbitrary laser mode. The match-filter, used to perform the azimuthal decomposition, is bounded by an annular ring, allowing us to conduct a scale-independent decomposition on our selected mode. This technique therefore requires no prior knowledge of the mode structure, the mode phases, or the amplitude distribution. A basis comprising of the angular harmonics is used to express the spatial distribution of the selected mode in terms of spatially dependant coefficients. We use this to infer directly from the measured weightings of the azimuthally decomposed modes and their phase-delay measurements, the intensity of the selected field, its phase, and its orbital angular momentum (OAM) density. We illustrate the concept by executing a full decomposition of two examples: a superposition of two Bessel beams, with relative phase differences, and an off-axis vortex mode. We show a reconstruction of the amplitude, phase and OAM density of these fields with a high degree of accuracy.
\end{abstract}

Keywords: Azimuthal decomposition, phase delays, Bessel beams, orbital angular momentum, field reconstruction

\section{INTRODUCTION}

Many complicated techniques have been applied to the problem of studying the structure and propagation characteristics of laser beams ${ }^{1-4}$. Recently, computer generated holograms, implemented in a mode multiplexing scheme, have been used for the modal decomposition of laser beams from fibres ${ }^{5,6}$. This approach has its disadvantages if one wishes to study a completely unknown laser source, as one requires information on the modal basis that is being measured, as well as the scale parameters of the basis.

In this paper we illustrate that without any knowledge of the mode structure, the mode phases, nor the scale of the amplitude distribution, we can perform a modal decomposition of an arbitrary laser source by making use of a basis comprising of angular harmonics ${ }^{7}$. From the measurement technique presented in this paper, we can infer directly the intensity of the superposition field, its phase, and its OAM density distribution. We illustrate the concept by executing a full modal decomposition of a superposition of two Bessel beams, with relative phase differences, and an off-axis vortex mode.

\section{CONCEPT AND THEORY}

An arbitrary optical field, $u(r, \phi)$, is expanded into a basis that is not dependent on scale, such as angular harmonics, $\exp (i l \varphi)$, that are orthogonal over the azimuthal plane

$$
u(r, \phi)=\frac{1}{\sqrt{2 \pi}} \sum_{l} a_{l}(r) \exp \left(i \Delta \theta_{l}\right) \exp (i l \phi),
$$

with the weighting coefficients given by

$$
a_{l}(r)=\frac{1}{\sqrt{2 \pi}} \int_{0}^{2 \pi} u(r, \phi) \exp \left(i \Delta \theta_{l}\right) \exp (-i l \phi) d \phi
$$

*adudley@csir.co.za; phone+27 (0)128413741

Complex Light and Optical Forces VII, edited by Jesper Glückstad, David L. Andrews, Enrique J. Galvez, Proc. of SPIE Vol. 8637, 86370D - (C) 2013 SPIE · CCC code: 0277-786X/13/\$18 · doi: 10.1117/12.2004252 
The modes therefore have real coefficients that contain information on the spatial distribution of the field, while each mode is allowed an arbitrary phase shift, $\Delta \theta_{l}$, relative to some reference, which we may take to be an external source or for convenience the first mode in the series, $l=0$. To completely describe $u(r, \phi$, ) the weighting coefficients are found by an inner product calculation with a suitable match filter, as shown in Eq. (2). To physically implement the inner-product, consider the signal at the origin of the Fourier plane after the field has been modulated by a transmission function given by $t_{l}(r, \phi)$

$$
U_{l}(0)=\frac{\exp (i 2 k f)}{i \lambda f} \int_{0}^{\infty} \int_{0}^{2 \pi} t_{l}(r, \phi) u(r, \phi) r d r d \phi
$$

In order to execute Eq. (2), the match filter has two requirements: (i) it should have an azimuthal phase variation opposite to the mode being investigated, and (ii) it consists of an annular slit centered at $r=R$ (of negligible thickness, $\Delta R$ ). Such a match filter is defined as

$$
t_{l}(r, \phi)=\left\{\begin{array}{cc}
\exp (-i l \phi) & R-\Delta R / 2<r<R+\Delta R / 2 \\
0 & \text { otherwise }
\end{array} .\right.
$$

The subscript $l$ in $t_{l}$ and $U_{l}$ refer to selecting the $l^{\text {th }}$ mode in the expansion of Eq. (1). So that

$$
\begin{aligned}
U_{l}(R, 0) & =\frac{\exp (i 2 k f)}{i \lambda f} \int_{0}^{\infty} \int_{0}^{2 \pi} t_{l}(r, \phi) u(r, \phi) r d r d \phi \\
& =\frac{\exp (i 2 k f)}{i \lambda f} \int_{R-\frac{\Delta R}{2}}^{R+\frac{\Delta R}{2}} \int_{0}^{2 \pi} a_{l}(r) \exp \left(i \Delta \theta_{l}\right) \exp (i l \phi) \exp (-i l \phi) r d r d \phi \\
& \approx \frac{k \exp (i 2 k f)}{i f} a_{l}(R) \exp \left(i \Delta \theta_{l}\right) R \Delta R .
\end{aligned}
$$

Re-arranging the final result in Eq. (5) reveals that

$$
a_{l}(R)=\frac{i f}{R \Delta R \cdot k \exp (i 2 k f)} U_{l}(R, 0) \exp \left(-i \Delta \theta_{l}\right) .
$$

The weighting coefficients are determined by a measurement of the intensity of the signal in the Fourier plane

$$
a_{l}(R)=\frac{f}{R \Delta R \cdot k} \sqrt{I_{l}(R, 0)} .
$$

By interfering the selected $l$ mode with a reference wave and considering the inner product signal $U_{l}(R, 0)$, the unknown phase term, $\Delta \theta_{l}$, can be found

$$
\begin{aligned}
I_{l}\left(\Delta \theta_{l}\right) & =\left|a_{l}(R) \exp \left(i \Delta \theta_{l}\right)+g\right|^{2} \\
& =a_{l}^{2}(R)+g^{2}+2 a_{l}(R) g \cos \left(\Delta \theta_{l}\right),
\end{aligned}
$$

where $g$ represents such a reference wave. The unknown phase delay between the modes is determined by inverting Eq. (8). With the complete modal decomposition of the field achieved, it is possible to calculate the intensity of the field, $|u(r, \phi)|^{2}$, the phase of the field, $\arg (u(r, \phi))$, the Poynting vector,

$$
\vec{S}=\frac{\varepsilon_{0} \omega c^{2}}{4}\left(i\left(u \nabla u^{*}-u^{*} \nabla u\right)+2 k|u|^{2} \hat{z}\right),
$$

and the OAM density

$$
L_{z}=\frac{1}{c^{2}}(r \times S)_{z} .
$$




\section{EXPERIMENTAL METHODOLOGY}

The experimental setup is depicted in Fig. 1 (a) where a HeNe laser was expanded through a $5 \times$ telescope and directed onto the liquid crystal display (LCD) of a spatial light modulator (SLM) (HoloEye, PLUTO-VIS, with 1920×1080 pixels of pitch $8 \mu \mathrm{m}$ and calibrated for a $2 \pi$ phase shift at $\sim 633 \mathrm{~nm}$ ). The first SLM (denoted as LCD 1) was programmed to produce various superposition fields using the concept of Durnin's ring-slit ${ }^{8}$, but implemented digitally $y^{9,10}$.

To create a superposition of two non-diffracting beams of opposite handedness, the ring slit was programmed with two opposite azimuthal phases, and the resulting field Fourier transformed at the focal plane after L3. The phase pattern in the annular region was separated into two ring-slits, each possessing an azimuthal phase of equal order but opposite handedness (Fig. 1(b)). When the orders, $|l|$, of the two azimuthal phases were of equal but of opposite handedness, a 'petal'-structure was produced, where the number of 'petals' is denoted by $2|l|^{11,12}$ (Fig. 1(c)).

The resulting superposition field was then magnified with a 10× objective and directed to the second SLM (LCD 2) for executing the modal decomposition. This was accomplished by executing an inner product of the incoming field with the match filter set to $\exp (i l \varphi)$, for various $l$ values, and for particular radial $(r)$ positions (Fig. 1(d)). There are two important aspects of this experiment: firstly, the detection is restricted to the origin of the resulting field after the Fourier transforming lens L4, and secondly, the $r$ dependence of the coefficient $a_{l}$ is found by implementing the match filter in the form of a narrow (20 pixels) annular ring of radius $r$.

(a)

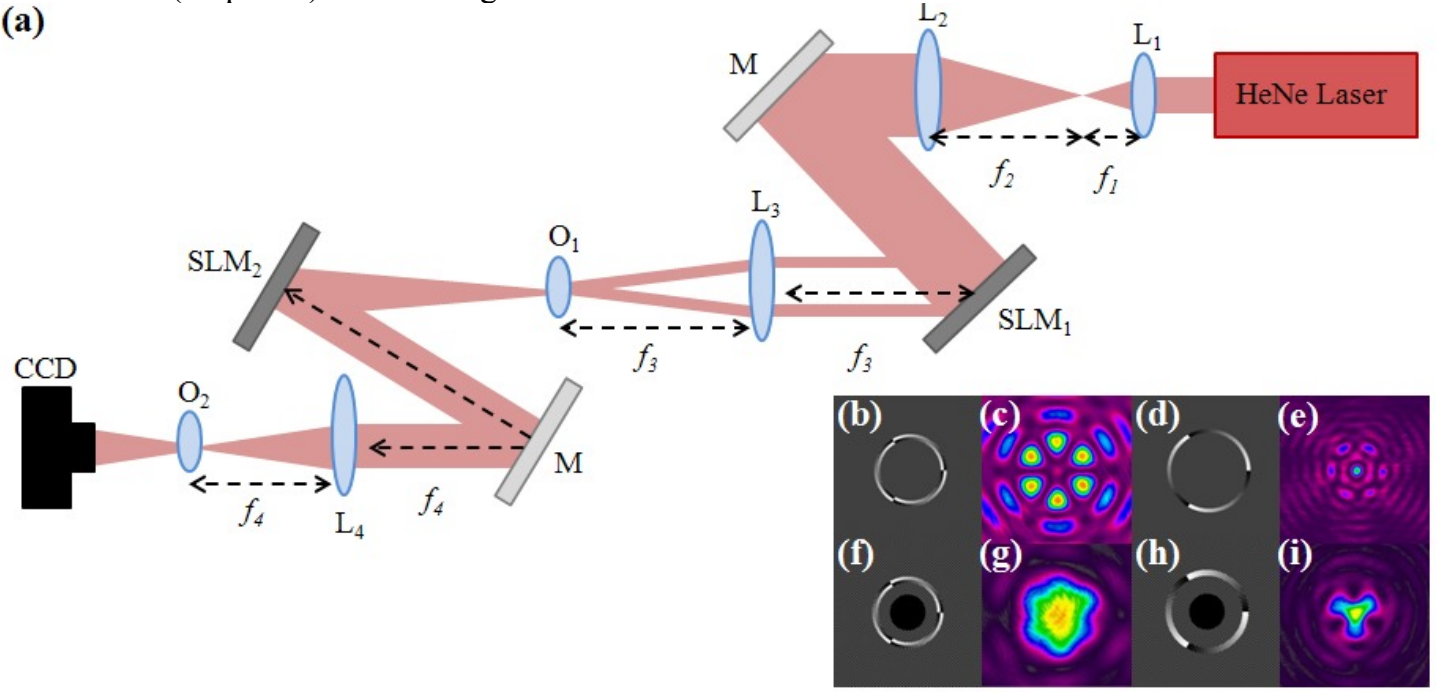

Fig. 1. (a) A schematic of the experimental setup for performing the modal decomposition of our superposition modes as a function of both $r$ and $l$. L: Lens $\left(\mathrm{f}_{1}=15 \mathrm{~mm} ; \mathrm{f}_{2}=75 \mathrm{~mm} ; \mathrm{f}_{3}=200 \mathrm{~mm}\right.$ and $\left.\mathrm{f}_{4}=200 \mathrm{~mm}\right)$; M: Mirror; SLM: Spatial light Modulator; O: Objective; CCD: CCD Camera. The objective, $\mathrm{O}_{2}$, was placed at the focus (or Fourier plane) of lens, $\mathrm{L}_{4}$. (b) The digital hologram used to generate the optical field of interest (c) and the digital hologram (d) used to extract the weightings of the modes from the inner product (e). The digital holograms for generating (f) and decomposing (h) the field, to extract the intermodal phase. The intensity profile of the field at the plane of $\mathrm{SLM}_{2}(\mathrm{~g})$ and CCD (i).

By switching off the checkerboard pattern in the centre of both SLMs (Figs 1 (f) and (h)), a portion of the initial Gaussian beam passes through the entire optical system, and can be used as the reference beam, $g$, in order to measure the phase delay between the modes. Changing between the intensity measurement (Fig. 1 (b) - (e)) and the phase measurement (Fig. 1 (f) - (i)) requires only a change in the holograms loaded to SLMs 1 and 2, which can be done at 60 $\mathrm{Hz}$, i.e., practically real-time acquisition of the necessary data.

\section{RESULTS AND DISCUSSION}

We first created a superposition of Bessel beams with opposite handedness, i.e., $m=3$ and $n=-3$, defined generally as

$$
u(r, \phi)=A_{m} J_{m}(k r) \exp (i m \phi)+A_{n} J_{n}(k r) \exp (i n \phi) \exp (i \Delta \theta) .
$$


The phase delay for the azimuthal mode of $-3, \Delta \theta$, was varied between 0 and $2 \pi$, resulting in a rotation of the intensity pattern illustrated in Fig 2 (a). The rotation was measured by observing the angular displacement of any one of the 'petals', which is directly related to the phase delay, thus allowing the measured phase shift to be compared to the programmed value. The result of this calibration test is given in Fig 2. (b), where it is clear that the agreement between measured and actual is very good (slope difference of $0.1 \%$ ). These results confirm that the setup was working correctly without spurious effects.

(a)

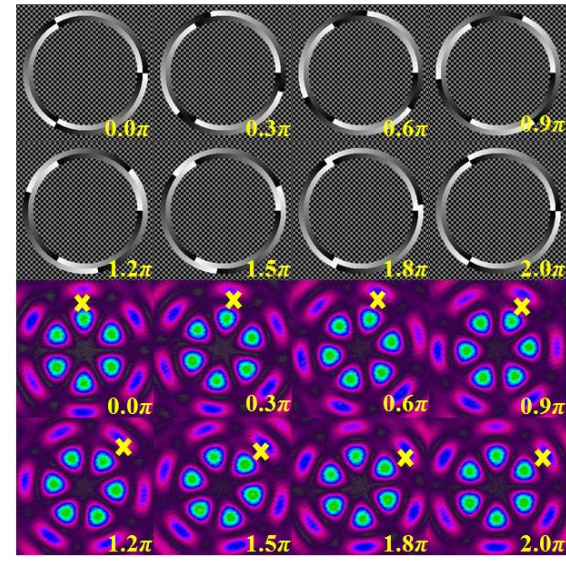

(b)

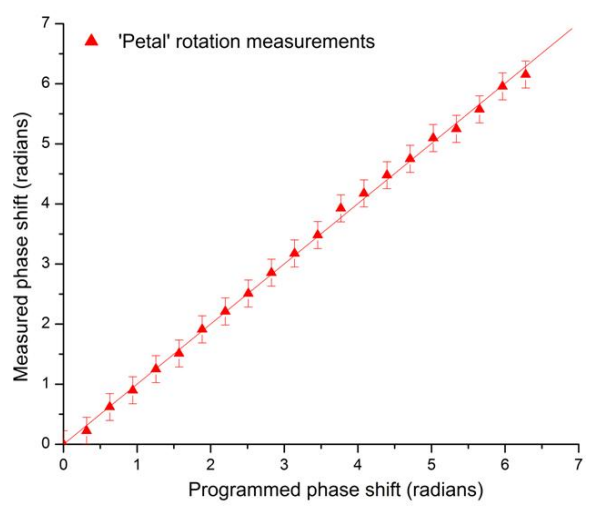

Fig. 2. (a) If the phase is shifted in one of the modes in the initial field (top two rows), then the measured 'petal' structure of the superposition field (bottom two rows) is seen to rotate. (b) Comparison of the measured phase shift to the actual phase shift.

The programmed phase shift $\Delta \theta$ was measured using the interference technique and determined by inverting Eq. (8). The phase shift in the $n=-3$ mode was programmed through a range of phase values from 0 to $2 \pi$ and the interference pattern was monitored, as illustrated in Fig. 3 (a). The offset phase of the reference beam was removed and the measured phase was then compared to the programmed phase, shown in Fig. 3 (b), and again the agreement is good (slope difference of $3 \%$ ). There are some uncertainties near a programmed phase shifts of $0.60 \pi$ and $1.60 \pi$, due to the flatness of the change in the measured intensity
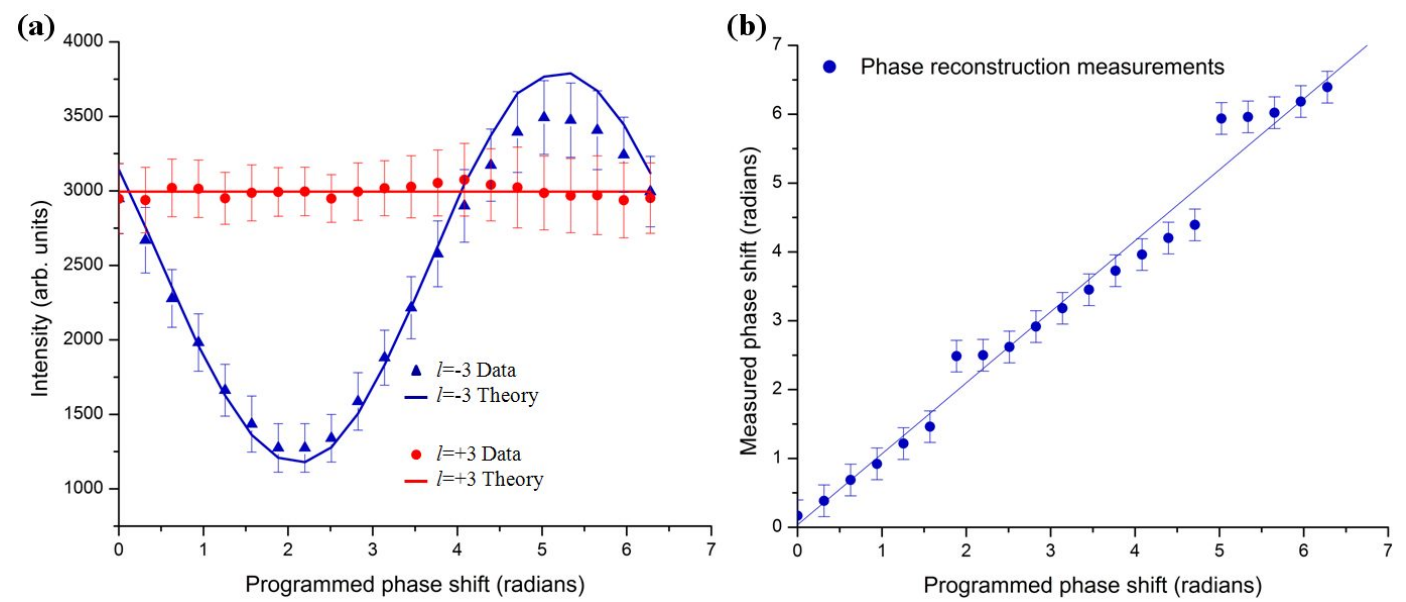

Fig. 3. (a) The interference of the modes with a reference wave results in a changing intensity at the origin of the Fourier plane, which can be used to infer the phase shift per mode; (b) comparison of the measured phase shift to the actual phase shift.

Next we deliberately broke the symmetry of the field by constructing a superposition field with $m=+3$ and $n=-2$. The annular ring on $\mathrm{SLM}_{2}$, of width $80 \mu \mathrm{m}$, was scanned through 28 radial positions and the azimuthal phase varied across a wide spectrum of modes. The only non-zero components were those corresponding to $l=+3$ and $l=-2$. For 
each ring position the unknown mode delay was computed. With all the unknown terms in Eq. (1) measured experimentally, we were able to reconstruct the intensity (Fig. 4 (b)) and phase (Fig. 4 (d)) of the initial field, and compare it to the theoretical prediction (Figs 4 (a) and (c), respectively). There is very good agreement between the theoretically predicted field and the field reconstructed from the modal decomposition.
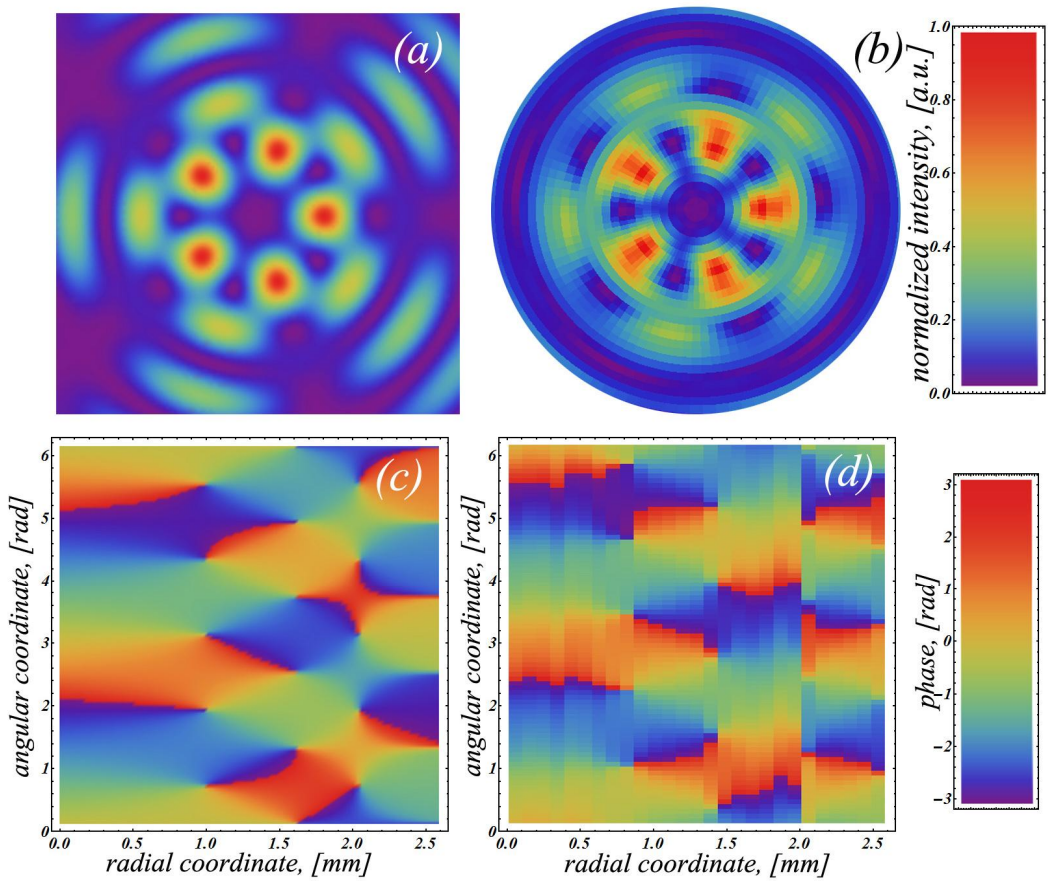

Fig. 4. Comparison of: (a) the theoretical, and (b) the experimentally reconstructed intensity profiles of the field, together with (c) the theoretical, and (d) the experimentally reconstructed phase profiles of the field.

We can also infer the OAM density of any arbitrary field directly from our measurement technique by the use of Eq. (10). The theoretical and experimental results of this are shown in Fig. 5 (a) and (b), respectively. We have previously performed such measurements without the modal phase information, and found successful agreement for particular optical fields ${ }^{13,14}$.
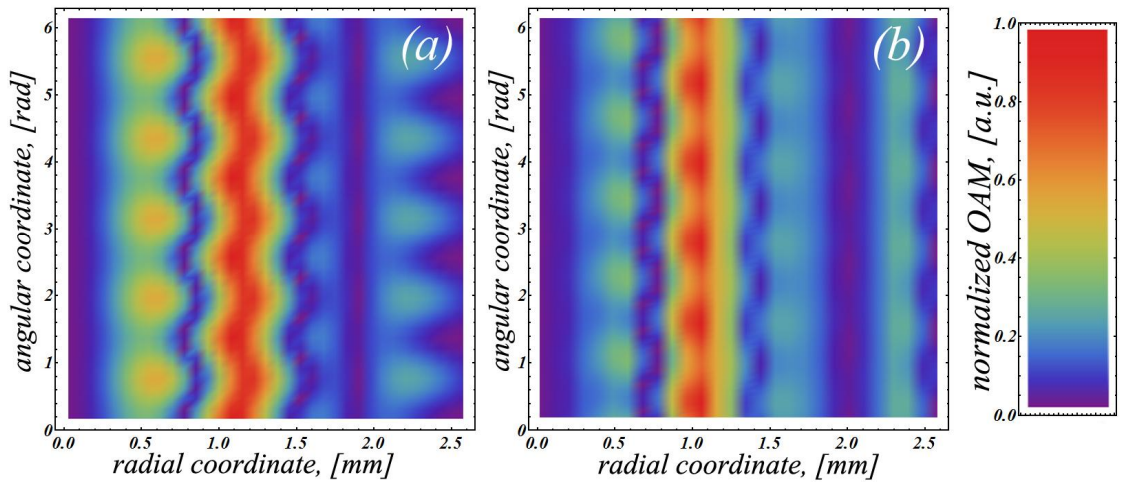

Fig. 5. The (a) theoretical and (b) experimental OAM density of the field.

To illustrate even further that this technique can be applied to any optical field, an example of an off-axis vortex was also studied and is present in Fig. 6. Our measurement technique was implemented on the field, given in Fig 6 (a) and the reconstructed field is shown in Fig. 6 (b), illustrating that this technique is successful in the reconstruction of optical fields which posses an on-axis intensity. Since there is no dark core in the centre of the field for which we could pass a Gaussian reference beam through, we brought a reference beam from another section. 


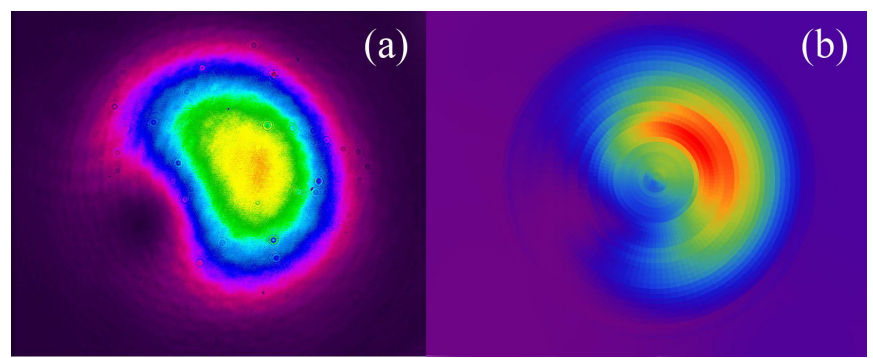

Fig. 6. A comparison of the experimentally recorded intensity (a) and the reconstructed intensity (b), for the off-axis vortex case.

\section{CONCLUSION}

We have outlined a simple technique for performing a complete azimuthal decomposition of an arbitrary laser mode that requires no scale information of the basis functions, and allows for the modal phases to be easily measured. We illustrated the concept by executing a full decomposition of two examples: a superposition of two Bessel beams, with relative phase differences, and an off-axis vortex mode and reconstructed the amplitude, phase and OAM density of these fields with a high degree of accuracy.

\section{REFERENCES}

[1] Tervonen, E., Turunen, J. and Friberg, A., "Transverse laser mode structure determination from spatial coherence measurements: experimental results," Appl. Phys. B 49, 409-414 (1989).

[2] Cutolo, A., Isernia, T., Izzo, I., Pierri, R. and Zerni, L., "Transverse mode analysis of a laser beam by near- and far-field intensity measurements," Appl. Opt. B 34, 7974-7978 (1995).

[3] Santarsiero, M., Gori, F., Borghi, R. and Guattari, G., "Evaluation of the modal structure of light beams composed of incoherent mixtures of Hermite-Gaussian modes," Appl. Opt. B 38, 5272-5281 (1999).

[4] Xue, X., Wei, H. and Kirk, A. G., "Intensity-based modal decomposition of optical beams in terms of HermiteGaussian functions," J. Opt. Soc. Am. A 17, 1086-1091 (2000).

[5] Flamm, D., Schmidt, O. A., Schulze, C., Borchardt, J., Kaiser, T., Schroter, S. and Duparre, M., "Measuring the spatial polarization distribution of multimode beams emerging from passive step-index large-mode-area fibers," Opt. Lett. 35(20), 3429-3431 (2010).

[6] Kaiser, T., Flamm, D., Schroter, S. and Duparre, M., "Complete modal decomposition for optical fibers using CGH-based correlation filters," Opt. Express 17(11), 9347-9356 (2009).

[7] Litvin, I., Dudley, A., Roux, F. S. and Forbes, A., "Azimuthal decomposition with digital holograms," Opt. Express 20(10), 10996-11004 (2012).

[8] Durnin, J., Miceli, J. J. and Eberly, J. H., “Diffraction-free beams," Phys. Rev. Lett. 58(15), 1499-1501 (1987).

[9] Rop, R., Dudley, A., Lopez-Mariscal, C. and Forbes, A., "Measuring the rotation rates of superpositions of higher-order Bessel beams," J. Mod. Opt. 59(3), 259-267 (2012).

[10] López-Mariscal, C. and Helmerson, K., "Shaped nondiffracting beams," Opt. Lett. 35(8), 1215-1217 (2010).

[11] Vasilyeu, R., Dudley, A., Khilo, N. and Forbes, A., "Generating superpositions of higher-order Bessel beams," Opt. Express 17(26), 23389-23395 (2009).

[12] Rop, R., Litvin, I. A. and Forbes, A., "Generation and propagation dynamics of obstructed and unobstructed rotating orbital angular momentum-carrying Helicon beams," J. Opt. 14(3), 035702 (2012).

[13]Litvin, I. A., Dudley, A. and Forbes, A., "Poynting vector and orbital angular momentum density of superpositions of Bessel beams," Opt. Express 19(18), 16760-16771 (2011).

[14] Dudley, A., Litvin, I. A. and Forbes, A., "Quantitative measurement of the orbital angular momentum density of light," Applied Optics 51(7), 823-833 (2012). 